PROCEEDINGS OF THE

AMERICAN MATHEMATICAL SOCIETY

Volume 140, Number 5, May 2012, Pages 1623-1631

S 0002-9939(2011)11001-3

Article electronically published on August 18, 2011

\title{
TANGENT BUNDLES TO REGULAR BASIC SETS IN HYPERBOLIC DYNAMICS
}

\author{
LUCHEZAR STOYANOV
}

(Communicated by Bryna Kra)

\begin{abstract}
Given a locally maximal compact invariant hyperbolic set $\Lambda$ for a $C^{2}$ flow or diffeomorphism on a Riemann manifold with $C^{1}$ stable laminations, we construct an invariant continuous bundle of tangent vectors to local unstable manifolds that locally approximates $\Lambda$ in a certain way.
\end{abstract}

\section{INTRODUCTION}

Let $M$ be a $C^{2}$ complete (not necessarily compact) Riemann manifold $M$ and let $\Lambda$ be a compact basic set for a $C^{2}$ flow $\phi_{t}: M \longrightarrow M(t \in \mathbb{R})$ or a $C^{2}$ diffeomorphism $f: U \longrightarrow f(U) \subset M$, where $U$ is an open neighbourhood of $\Lambda$ in $M$. Let $E^{u}(x)$ and $E^{s}(x)(x \in \Lambda)$ be the tangent spaces to the local stable and unstable manifolds $W_{\epsilon}^{s}(x)$ and $W_{\epsilon}^{u}(x)$ of size $\epsilon>0$, respectively (see Section 2). We say that $\phi_{t}$ has $C^{1}$ stable laminations over $\Lambda$ if the map $\Lambda \ni x \mapsto E^{s}(x)$ is $C^{1}$; i.e. at each $x \in \Lambda$ this map has a linearization that depends continuously on $x$.

Given $x \in \Lambda$, let $k_{x} \geq 1$ be the minimal integer such that there exist $\delta_{x} \in(0, \epsilon)$ and a $k_{x}$-dimensional $C^{1}$ submanifold $S_{x}$ of $W_{\epsilon}^{u}(x)$ such that $\Lambda \cap W_{\delta_{x}}^{u}(x) \subset S_{x}$. We will then say that $S_{x}$ is a $u$-submanifold of minimal dimension at $x$ and size $\delta_{x}$. In general $S_{x}$ is not (locally) unique; however it turns out that, under certain regularity conditions, its tangent space $T_{x} S_{x}$ is uniquely determined, invariant and continuous:

Theorem 1.1. Assume that $\Lambda$ is a compact basic set for the $C^{2}$ flow $\phi_{t}$ (or the $C^{2}$ diffeomorphism $f$ ) such that the local stable laminations over $\Lambda$ are $C^{1}$ and $\phi_{t}$ is topologically mixing on $\Lambda$. Then there exists an integer $k=k^{u} \geq 1$ and a continuous $d \phi_{t}$-invariant (or $d f$-invariant, respectively) distribution $E_{\Lambda}^{u}(x), x \in \Lambda$, of $k$-dimensional linear spaces such that for any $x \in \Lambda$ we have $k_{x}=k, E_{\Lambda}^{u}(x) \subset$ $E^{u}(x)$ and $T_{x} S_{x}=E_{\Lambda}^{u}(x)$ for any u-submanifold $S_{x}$ of minimal dimension at $x$. Moreover, the distribution $E_{\Lambda}^{u}$ is invariant under the linearizations of the local stable holonomy maps as well.

We refer the reader to Section 2 for the definition of the local stable holonomy maps.

Received by the editors July 28, 2010 and, in revised form, November 27, 2010 and January 10, 2011

2010 Mathematics Subject Classification. Primary 37D20, 37D40.

The author thanks the referee for useful comments and suggestions.

(C)2011 American Mathematical Society 
In a similar way one defines $s$-submanifolds of minimal dimension at $x \in \Lambda$, and a result similar to the above holds for these.

Remark 1.2. It is easy to see that all vectors in $E^{u}(x)$ that are tangent to the basic set $\Lambda$ belong to $E_{\Lambda}^{u}(x)$. However, in general the span of these tangent vectors could be a proper subspace of $E_{\Lambda}^{u}(x)$; see Example 4.2 below.

The motivation for the introduction of the bundles $E_{\Lambda}^{u, s}$ comes from [St2] (see also St4]) which deals with Dolgopyat-type spectral estimates ([D]) for Axiom A flows on basic sets, where one has to take into account some fine geometric properties of basic sets. As it turns out there, in certain cases when the basic set $\Lambda$ is 'relatively small', considering the tangent maps $d \phi_{t}$ over the whole bundle $E^{u}$ (or $E^{s}$ ) does not say enough about the dynamics of the flow near $\Lambda$. So, it appears, restricting $d \phi_{t}$ over the smallest possible invariant subbundle of $E^{u}$ would be beneficiall 1 and it is natural to believe that this might be the case in some other situations, as well, particularly when the dimension of the spaces $E^{u}(x)$ (or $E^{s}(x)$ ) is large.

In Section 3 below we prove Theorem 1.1 for flows; for diffeomorphisms the argument is very similar. Some basic definitions are given in Section 2, while Section 4 contains two examples.

\section{Preliminaries}

Let $M$ be a $C^{2}$ complete (not necessarily compact) Riemann manifold, and let $\phi_{t}: M \longrightarrow M(t \in \mathbb{R})$ be a $C^{2}$ flow on $M$. So, in particular $\phi_{t}: M \longrightarrow M$ is a diffeomorphism for all $t \in \mathbb{R}$ and $\phi_{t} \circ \phi_{s}=\phi_{s+t}$ for all $t, s \in \mathbb{R}$. A $\phi_{t^{-}}$ invariant closed subset $\Lambda$ of $M$ is called hyperbolic if $\Lambda$ contains no fixed points and there exist constants $C>0$ and $0<\lambda<1$ and a $d \phi_{t}$-invariant decomposition $T_{x} M=E^{0}(x) \oplus E^{u}(x) \oplus E^{s}(x)$ of $T_{x} M(x \in \Lambda)$ into a direct sum of non-zero linear subspaces, where $E^{0}(x)$ is the one-dimensional subspace determined by the direction of the flow at $x,\left\|d \phi_{t}(u)\right\| \leq C \lambda^{t}\|u\|$ for all $u \in E^{s}(x)$ and $t \geq 0$, and $\left\|d \phi_{t}(u)\right\| \leq C \lambda^{-t}\|u\|$ for all $u \in E^{u}(x)$ and $t \leq 0$. Here $\|\cdot\|$ is the norm on $T_{x} M$ determined by the Riemann metric on $M$. See e.g. Chapters 6, 18 and 19 in $[\mathrm{KH}]$ for general information on hyperbolic sets.

A non-empty compact $\phi_{t}$-invariant hyperbolic subset $\Lambda$ of $M$ which is not a single closed orbit is called a basic set for $\phi_{t}$ if $\phi_{t}$ is transitive on $\Lambda$ and $\Lambda$ is locally maximal, i.e. there exists an open neighbourhood $V$ of $\Lambda$ in $M$ such that $\Lambda=\bigcap_{t \in \mathbb{R}} \phi_{t}(V)$. We should emphasize that the basic sets $\Lambda$ considered in this article are always assumed to be compact, and we also assume that the flow $\phi_{t}$ is topologically mixing on $\Lambda$ (see e.g. Section 4 in [Ch] or Section 1.8 and Chapter 18 in $[\mathrm{KH}]$ for general information concerning topologically mixing flows).

From now on we will assume that $\Lambda$ is a basic set for the flow $\phi_{t}$. For $x \in \Lambda$ and a sufficiently small $\epsilon>0$ let

$$
\begin{aligned}
& W_{\epsilon}^{s}(x)=\left\{y \in M: d\left(\phi_{t}(x), \phi_{t}(y)\right) \leq \epsilon \text { for all } t \geq 0, d\left(\phi_{t}(x), \phi_{t}(y)\right) \rightarrow_{t \rightarrow \infty} 0\right\}, \\
& W_{\epsilon}^{u}(x)=\left\{y \in M: d\left(\phi_{t}(x), \phi_{t}(y)\right) \leq \epsilon \text { for all } t \leq 0, d\left(\phi_{t}(x), \phi_{t}(y)\right) \rightarrow_{t \rightarrow-\infty} 0\right\}
\end{aligned}
$$

be the (strong) stable and unstable manifolds of size $\epsilon$ (see e.g. Section $6.4 \mathrm{in}[\mathrm{KH}]$ ). Then $E^{u}(x)=T_{x} W_{\epsilon}^{u}(x)$ and $E^{s}(x)=T_{x} W_{\epsilon}^{s}(x)$.

Assuming $\epsilon_{1} \in\left(0, \epsilon_{0}\right]$ is sufficiently small, for any $x \in \Lambda$ and $y \in \Lambda \cap W_{\epsilon_{1}}^{s}(x)$ the local holonomy map $\mathcal{H}_{x}^{y}: \Lambda \cap W_{\epsilon_{1}}^{u}(x) \longrightarrow \Lambda \cap W_{\epsilon_{0}}^{u}(y)$ along stable laminations

\footnotetext{
${ }^{1}$ Although in St4 we found a way around the problem by different means.
} 
is well-defined and uniformly Hölder continuous (see e.g. [PSW]). Recall that the map $\mathcal{H}_{x}^{y}$ is defined as follows. Given $z \in \Lambda \cap W_{\epsilon_{1}}^{u}(x)$, there exist unique $z^{\prime} \in W_{\epsilon_{1}}^{s}(z)$ and $y^{\prime} \in W_{\epsilon_{0}}^{u}(y)$ such that $\phi_{t}\left(z^{\prime}\right)=y^{\prime}$ for some $t \in \mathbb{R},|t| \leq \epsilon_{0}$. Then we set $\mathcal{H}_{x}^{y}(z)=y^{\prime}$. Under the additional condition that the stable laminations are $C^{1}$, the maps $\mathcal{H}_{x}^{y}$ are $C^{1}$ as well (see e.g. Fact (2) on p. 647 in [Ha]). That is, for each $z \in \Lambda \cap W_{\epsilon_{1}}^{u}(x)$ the map $\mathcal{H}_{x}^{y}$ has a linearization $L_{x}^{y}(z): E^{u}(z) \longrightarrow E^{u}\left(\mathcal{H}_{x}^{y}(z)\right)$ at $z \in \Lambda \cap W_{\epsilon_{1}}^{u}(x)$ and $\left\|L_{x}^{y}(z)\right\| \leq C$ for some constant $C>0$ independent of $x, y$ and $z$.

\section{Proof of Theorem 1.1}

Assume that $\phi_{t}: M \longrightarrow M(t \in \mathbb{R})$ is a $C^{2}$ flow on $M$ and $\Lambda$ is a compact basic set for $\phi_{t}$ such that the local stable laminations over $\Lambda$ are $C^{1}$ and $\phi_{t}$ is topologically mixing on $\Lambda$. Then, as mentioned in Section 2, assuming $0<\epsilon_{1} \leq \epsilon_{0}$ are sufficiently small, for any $x \in \Lambda$ and $y \in \Lambda \cap W_{\epsilon_{1}}^{s}(x)$ the local stable holonomy $\operatorname{map} \mathcal{H}_{x}^{y}: \Lambda \cap W_{\epsilon_{1}}^{u}(x) \longrightarrow \Lambda \cap W_{\epsilon_{0}}^{u}(y)$ is $C^{1}$.

By Whitney's Theorem (see e.g. Section I.3 in [M]), the map $\mathcal{H}_{x}^{y}$ has a $C^{1}$ extension $\widetilde{\mathcal{H}}_{x}^{y}: W_{\epsilon_{1}}^{u}(x) \longrightarrow W_{\epsilon_{0}}^{u}(y)$ with $\left\|d \widetilde{\mathcal{H}}_{x}^{y}\right\| \leq C$ on $W_{\epsilon_{1}}^{u}(x)$. Since

$$
\left\|\left(d \widetilde{\mathcal{H}}_{x}^{y}(x)\right)^{-1}\right\|=\left\|\left(d \mathcal{H}_{x}^{y}(x)\right)^{-1}\right\|=\left\|d \mathcal{H}_{y}^{x}(y)\right\| \leq C,
$$

it follows from the Inverse Function Theorem that there exists $\epsilon_{2} \in\left(0, \epsilon_{1}\right]$ such that

$$
\widetilde{\mathcal{H}}_{x}^{y}: W_{\epsilon_{2}}^{u}(x) \longrightarrow \widetilde{\mathcal{H}}_{x}^{y}\left(W_{\epsilon_{2}}^{u}(x)\right) \subset W_{\epsilon_{0}}^{u}(y)
$$

is a diffeomorphism for all $x \in \Lambda$ and all $y \in \Lambda \cap W_{\epsilon_{1}}^{s}(x)$.

Let $k_{x}(x \in \Lambda)$ be the integers introduced in Section 1 . Set $k=\min _{x \in \Lambda} k_{x}$, and fix an arbitrary $x_{0} \in \Lambda$ with $k_{x_{0}}=k$.

The $C^{1}$ regularity of the local stable laminations is needed in the following lemma. The rest of the proof of Theorem 1.1 does not use this assumption.

Lemma 3.1. We have $k_{x}=k$ for every $x \in \Lambda$. Moreover, there exist $\delta>0$ and for every $x \in \Lambda$ a k-dimensional $C^{1}$ submanifold $S_{x}$ of $W_{\delta}^{u}(x)$ such that $\Lambda \cap W_{\delta}^{u}(x) \subset$ $S_{x}$

Proof. First, notice that if $S_{x}$ is a $C^{1}$ submanifold of $W_{\delta}^{u}(x)$ of minimal dimension such that $\Lambda \cap W_{\delta}^{u}(x) \subset S_{x}$ for some $x \in \Lambda$, then for any $y \in \Lambda \cap W_{\epsilon_{1}}^{s}(x), \widetilde{\mathcal{H}}_{x}^{y}\left(S_{x}\right)$ is a $C^{1}$ submanifold of $W_{\epsilon_{0}}^{u}(y)$ with $\Lambda \cap W_{\delta / C}^{u}(y) \subset \widetilde{\mathcal{H}}_{x}^{y}\left(S_{x}\right)$, so $k_{y} \leq k_{x}$. Similarly, $k_{x} \leq k_{y}$, so $k_{x}=k_{y}$. Moreover, we can choose the same $\delta>0$ for all $y \in \Lambda \cap W_{\epsilon_{1}}^{s}(x)$.

Let $S=S_{x_{0}}$ be a $u$-submanifold of $W_{\delta}^{u}\left(x_{0}\right)$ of minimal dimension such that $\Lambda \cap W_{\delta}^{u}\left(x_{0}\right) \subset S$ for some $\delta>0$. Since $\phi_{t}$ is topologically mixing on $\Lambda$, given $z \in \Lambda$, for every sufficiently large $t_{0}>0$ there exists $t \geq t_{0}$ such that $\phi_{t}\left(\Lambda \cap W_{\delta / 2}^{u}\left(x_{0}\right)\right) \cap$ $W_{\epsilon_{1}}^{s}(z) \neq \emptyset$ (see e.g. Proposition 18.3.10 in $[\mathrm{KH}]$ for the analogous property in the case of diffeomorphisms; for flows the proof is similar). Take $t>0$ with this property so large that $\phi_{t}$ is expanding on $W_{\delta / 2}^{u}\left(x_{0}\right)$, i.e. $d\left(\phi_{t}(p), \phi_{t}(q)\right) \geq d(x, y)$ for all $p, q \in W_{\delta / 2}^{u}\left(x_{0}\right)$. Let $z^{\prime}=\phi_{t}(x) \in W_{\epsilon_{1}}^{s}(z)$ for some $x \in \Lambda \cap W_{\delta / 2}^{u}\left(x_{0}\right)$. Since $S_{x}=S \cap W_{\delta / 2}^{u}(x) \subset S \cap W_{\delta}^{u}\left(x_{0}\right), S_{x}$ contains $\Lambda \cap W_{\delta / 2}^{u}(x)$ and $\operatorname{dim}\left(S_{x}\right)=k \leq k_{x}$, it follows that $k_{x}=k$ and $S_{x}$ is a $u$-submanifold of minimal dimension at $x$ and size $\delta$.

It now follows that $k_{z^{\prime}}=k$ and, since $\phi_{t}$ is expanding on $W_{\delta / 2}^{u}(x)$, there exists a $u$-submanifold of minimal dimension at $z^{\prime}$ of size $\delta / 2$. The remark in the beginning 
of the proof now shows that $k_{z}=k_{z^{\prime}}=k$, and there exists a $u$-submanifold of minimal dimension at $z$ of size $\delta /(2 C)$.

Lemma 3.2. For any $x \in \Lambda$ and any two u-submanifolds $S$ and $S^{\prime}$ of minimal dimension at $x$ we have $T_{x} S=T_{x} S^{\prime}$.

Proof. Let $S$ and $S^{\prime}$ be $u$-submanifolds of minimal dimension $k$ at $x$ for some $x \in \Lambda$. Take $\delta>0$ so small that $\Lambda \cap W_{\delta}^{u}(x) \subset S$ and $\Lambda \cap W_{\delta}^{u}(x) \subset S^{\prime}$.

Assume that $T_{x} S \backslash T_{x} S^{\prime} \neq \emptyset$ and fix an arbitrary $v \in T_{x} S \backslash T_{x} S^{\prime}$. Then we must have $n=\operatorname{dim}\left(W_{\delta}^{u}(x)\right)>k$. Choosing a smaller $\delta>0$ if necessary and using an appropriate submanifold chart for $S^{\prime}$ about $x$ in $W_{\delta}^{u}(x)$, one constructs a $C^{1}$ submanifold $S^{\prime \prime}$ of $W_{\delta}^{u}(x)$ such that $\operatorname{dim}\left(S^{\prime \prime}\right)=n-1, S^{\prime} \subset S^{\prime \prime}$ and $v \notin T_{x} S^{\prime \prime}$. Then $T_{x} S+T_{x} S^{\prime \prime}=E^{u}(x)=T_{x}\left(W_{\delta}^{u}(x)\right)$, so the submanifolds $S$ and $S^{\prime \prime}$ are transversal at $x$ (see e.g. Section 1.5 in [GP]). Consequently, there exists $\delta^{\prime} \in(0, \delta]$ so that $S^{\prime \prime \prime}=S \cap S^{\prime \prime} \cap W_{\delta^{\prime}}^{u}(x)$ is a submanifold of $W_{\delta^{\prime}}^{u}(x)$ of dimension at most $k-1$. Since $\Lambda \cap W_{\delta^{\prime}}^{u}(x) \subset S^{\prime \prime \prime}$, this is a contradiction with the minimality of $k=k_{x}$.

Thus, we must have $T_{x} S \subset T_{x} S^{\prime}$ and similarly $T_{x} S^{\prime} \subset T_{x} S$.

Proof of Theorem 1.1. For any $x \in \Lambda$ define $E_{\Lambda}^{u}(x)=T_{x} S$, where $S$ is an arbitrary $u$-submanifold of minimal dimension at $x$. By Lemma 3.2, the definition is correct. Moreover, $E_{\Lambda}^{u}(x) \subset E^{u}(x)$ and $T_{x} S_{x}=E_{\Lambda}^{u}(x)$ for any $u$-submanifold $S_{x}$ of minimal dimension at $x$. The latter implies $E_{\Lambda}^{u}\left(\phi_{t}(x)\right)=d \phi_{t}(x) \cdot E_{\Lambda}^{u}(x)$ for any $t \in \mathbb{R}$ and any $x \in \Lambda$.

It remains to show that the map $\Phi: \Lambda \ni x \mapsto E_{\Lambda}^{u}(x)$ is continuous. Fix for a moment $z \in \Lambda$ and let $S_{z}$ be a $u$-submanifold of minimal dimension at $z$. Then for any $t \in \mathbb{R}$ with a sufficiently small $|t|, \phi_{t}\left(S_{z}\right)$ is a $u$-submanifold of minimal dimension at $z^{\prime}=\phi_{t}(z)$, so $E_{\Lambda}^{u}\left(z^{\prime}\right)=T_{z^{\prime}}\left(\phi_{t}\left(S_{z}\right)\right)=d \phi_{t}(z) \cdot\left(T_{z} S_{z}\right)$ depends continuously on $z^{\prime}=\phi_{t}(z)$. That is, $\Phi$ is continuous along the orbit of $z$.

Next, choosing a sufficiently small $\delta>0$, for $z^{\prime} \in W_{\delta}^{u}(z) \cap \Lambda$ we have $z^{\prime} \in S_{z}$, so $E_{\Lambda}^{u}\left(z^{\prime}\right)=T_{z^{\prime}} S_{z}$, and therefore $\Phi\left(z^{\prime}\right)=E_{\Lambda}^{u}\left(z^{\prime}\right)$ depends continuously on $z^{\prime} \in$ $W_{\delta}^{u}(z) \cap \Lambda$.

Finally, for $z^{\prime} \in W_{\epsilon_{1}}^{s}(z) \cap \Lambda, \widetilde{\mathcal{H}}_{z}^{z^{\prime}}\left(S_{z}\right)$ is a $u$-submanifold of minimal dimension at $z^{\prime}$, so $E_{\Lambda}^{u}\left(z^{\prime}\right)=d \widetilde{\mathcal{H}}_{z}^{z^{\prime}}(z) \cdot\left(E_{\Lambda}^{u}(z)\right)$, and therefore $\Phi\left(z^{\prime}\right)=E_{\Lambda}^{u}\left(z^{\prime}\right)$ depends continuously on $z^{\prime} \in W_{\epsilon_{1}}^{s}(z) \cap \Lambda$.

The above and the continuity of the local product near $z$ imply that $\Phi$ is continuous at $z$.

\section{EXAmples}

We use two very well-known kinds of flows to give 1) a non-trivial example where $k^{u}<\operatorname{dim}\left(E^{u}\right)$ and 2) an example where the span of the tangent vectors in $E^{u}(z)$ to $\Lambda$ at $z$ form a proper subspace of $E_{\Lambda}^{u}(z)$ (for any $z \in \Lambda$ ). We use open billiard flows for the first example and geodesic flows on hyperbolic manifolds for the second. In higher dimensions both kinds of flows should provide a great variety of nonwandering sets with complicated geometric (and metric) structures (see e.g [Ka]); however it appears they would not be easy to investigate. It is quite possible that one could use other models that are easier to describe to get similar examples.

There are various possible ways to define tangent vectors to a subset of a manifold. Here we use the most straightforward one. Given $z \in \Lambda$, let $\exp _{z}^{u}: E^{u}(z) \longrightarrow$ $W_{\epsilon_{0}}^{u}(z)$ be the corresponding exponential map. A vector $b \in E^{u}(z) \backslash\{0\}$ is called 
tangent to $\Lambda$ at $z$ if there exist infinite sequences $\left\{v^{(m)}\right\} \subset E^{u}(z)$ and $\left\{t_{m}\right\} \subset \mathbb{R} \backslash\{0\}$ such that $\exp _{z}^{u}\left(t_{m} v^{(m)}\right) \in \Lambda \cap W_{\epsilon}^{u}(z)$ for all $m, v^{(m)} \rightarrow b$ and $t_{m} \rightarrow 0$ as $m \rightarrow \infty$. It is easy to see that a vector $b \in E^{u}(z) \backslash\{0\}$ is tangent to $\Lambda$ at $z$ iff there exists a $C^{1}$ curve $z(t), 0 \leq t \leq a$, in $W_{\epsilon}^{u}(z)$ for some $a>0$ with $z(0)=z, \dot{z}(0)=b$, and $z(t) \in \Lambda$ for arbitrarily small $t>0$. Denote by $\widehat{E}^{u}(z)$ the set of all non-zero tangent vectors $v \in E^{u}(z)$ to $\Lambda$ at $z$. Tangent vectors to $\Lambda$ in $E^{s}(z)$ and the set $\widehat{E}^{s}(z)$ are defined similarly. Clearly the sets $\widehat{E}^{u, s}(z)$ are non-empty and $d \phi_{t}$-invariant and $\widehat{E}^{u, s}(z) \subset E_{\Lambda}^{u, s}(z)$. However in general the span of $\widehat{E}^{u, s}(z)$ does not coincide with $E_{\Lambda}^{u, s}(z)$; see Example 4.2 below.

A simple example when $k_{u}<\operatorname{dim}\left(E^{u}\right)$ is given by a product $M=M^{\prime} \times \mathbb{R}^{d}$, $f=f^{\prime} \times A$, where $f^{\prime}$ is an Axiom A diffeomorphism on a manifold $M^{\prime}$ and $A$ is a hyperbolic linear map. A similar simple example can be given for flows. We believe that not all examples are like that.

Open problem. Under the assumptions in Section 1, assume that $k_{u}<\operatorname{dim}\left(E^{u}(z)\right)$ (or $\left.k_{s}<\operatorname{dim}\left(E^{s}(z)\right)\right), z \in \Lambda$. Does there always exist a $C^{1}$ submanifold $M^{\prime}$ of $M$ of positive codimension and an open neighbourhood $U$ of $\Lambda$ in $M$ such that $\phi_{t}\left(U \cap M^{\prime}\right) \subset M^{\prime}$ for all $t \in \mathbb{R}\left(\right.$ resp. $f^{n}\left(U \cap M^{\prime}\right) \subset M^{\prime}$ for all $\left.n \in \mathbb{Z}\right)$ ?

One would expect the answer to be negative; however at this stage we do not have examples to prove this. Example 4.1 below is non-trivial in a certain sense and in fact might be good enough to answer the above question in the negative; however we do not go that far here.

4.1. Open billiard flows. Let $K$ be a subset of $\mathbb{R}^{n}(n \geq 2)$ of the form $K=$ $K_{1} \cup K_{2} \cup \ldots \cup K_{k_{0}}$, where $K_{i}$ are compact strictly convex disjoint domains in $\mathbb{R}^{n}$ with $C^{r}$ boundaries $\Gamma_{i}=\partial K_{i}(r \geq 2)$ and $k_{0} \geq 3$. Set $\Omega=\overline{\mathbb{R}^{n} \backslash K}$ and $\Gamma=\partial K$. We assume that $K$ satisfies the following no-eclipse condition: for every pair $K_{i}, K_{j}$ of different connected components of $K$ the convex hull of $K_{i} \cup K_{j}$ has no common points with any other connected component of $K$. With this condition, the billiard flow $\phi_{t}$ defined on the cosphere bundle $S^{*}(\Omega)$ in the standard way is called an open billiard flow. It has singularities; however its restriction to the non-wandering set $\Lambda$ (the set of those $x \in S^{*}(\Omega)$ such that the trajectory $\left\{\phi_{t}(x): t \in \mathbb{R}\right\}$ is bounded) has only simple discontinuities at reflection points 2 Moreover, $\Lambda$ is compact, $\phi_{t}$ is hyperbolic and transitive on $\Lambda$, and it follows from St1] that $\phi_{t}$ is non-lattice and therefore by a result of Bowen $[\mathrm{B}$ it is topologically mixing on $\Lambda$. Finally, it follows from [St3 that when the minimal distance between distinct connected components of $K$ is relatively large compared to the maximal sectional curvature of $\partial K$, the open billiard flow on $\Lambda$ satisfies a pinching condition which implies that the stable laminations over $\Lambda$ are $C^{1}$.

In the following example we will use the natural symbolic coding of the open billiard. Let $A$ be the $k_{0} \times k_{0}$ matrix with entries $A(i, j)=1$ if $i \neq j$ and $A(i, i)=0$

\footnotetext{
${ }^{2}$ Notice that the natural projection of $\phi_{t}$ on the quotient space $S^{*}(\Omega) / \sim$, where $\sim$ is the equivalence relation $(q, v) \sim(p, w)$ iff $q=p$ and $v=w$ or $q=p \in \partial K$ and $v$ and $w$ are symmetric with respect to $T_{q}(\partial K)$, is continuous. Moreover whenever both $x$ and $\phi_{t}(x)$ are in the interior of $S^{*}(\Omega)$ and sufficiently close to $\Lambda$, the map $y \mapsto \phi_{t}(y)$ is smooth on a neighbourhood of $x$. It follows from well-known results of Sinai that $\Lambda$ is a hyperbolic set for $\phi_{t}$, and it is easily seen that $\Lambda$ is the maximal compact $\phi_{t}$-invariant subset of $S^{*}(\Omega)$. Moreover, it follows from the natural symbolic coding for the natural section of the flow that the periodic points are dense in $\Lambda$ and $\phi_{t}$ is transitive on $\Lambda$. Thus, $\Lambda$ is a basic set for $\phi_{t}$ and the classical theory of hyperbolic flows applies (see e.g. Part 4 in $[\mathrm{KH}]$ ).
} 
for all $i$, and let $\Sigma_{A}$ be the set of all sequences $\eta=\left(\eta_{j}\right)_{j=-\infty}^{\infty}$ of integer numbers $1 \leq \eta_{j} \leq k_{0}$ such that $\eta_{j} \neq \eta_{j+1}$ for all $j \in \mathbb{Z}$. Let $\operatorname{pr}_{1}: S^{*}(\Omega) \longrightarrow \Omega$ be the natural projection. Given $\xi \in \Sigma_{A}$, let $\left(P_{j}(\xi)\right)_{j=-\infty}^{\infty}$ be the successive reflection points of the unique billiard trajectory in the exterior of $K$ such that $P_{j}(\xi) \in$ $K_{\xi_{j}}$ for all $j \in \mathbb{Z}$ (see e.g. [St1]). Define the map $\Phi: \Sigma_{A} \longrightarrow \Lambda \cap S_{\partial K}^{*}(\Omega)$ by $\Phi(\xi)=\left(P_{0}(\xi),\left(P_{1}(\xi)-P_{0}(\xi)\right) /\left\|P_{1}(\xi)-P_{0}(\xi)\right\|\right)$. Then $\Phi$ is a bijection such that $\Phi \circ \sigma=B \circ \Phi$, where $B: \Lambda \cap S_{\partial K}^{*}(\Omega) \longrightarrow \Lambda \cap S_{\partial K}^{*}(\Omega)$ is the billiard ball map from boundary to boundary and $\sigma$ is the shift map on $\Sigma_{A}$.

Example 4.1. Assume that $n=3$ and there exists a plane $\alpha$ such that each of the domains $K_{j}$ is symmetric with respect to $\alpha$. Setting $K^{\prime}=K \cap \alpha$ and $\Omega^{\prime}=\Omega \cap \alpha$, it is easy to observe that every billiard trajectory generated by a point in $\Lambda$ is entirely contained in $\alpha$. That is, $\Lambda=\Lambda^{\prime}$, where $\Lambda^{\prime}$ is the non-wandering set for the open billiard flow in $\Omega^{\prime}$. Thus, $\operatorname{dim}\left(E_{\Lambda}^{u}(z)\right)=1<\operatorname{dim}\left(E^{u}(z)\right)=2$ for any $z \in \Lambda$. This example is of course trivial, since $\Lambda$ is contained in the flow-invariant submanifold $S^{*}\left(\Omega^{\prime}\right)$ of $S^{*}(\Omega)$.

However with a small local perturbation of the boundary $\partial K$ of $K$ we can get a non-trivial example. Choosing standard Cartesian coordinates $x, y, z$ in $\mathbb{R}^{3}$, we may assume that $\alpha$ is given by the equation $z=0$, i.e. $\alpha=\mathbb{R}^{2} \times\{0\}$. Let $\operatorname{pr}_{1}: S^{*}\left(\mathbb{R}^{3}\right) \sim \mathbb{R}^{3} \times \mathbb{S}^{2} \longrightarrow \mathbb{R}^{3}$ be the natural projection, and let $C=\operatorname{pr}_{1}(\Lambda)$. We may choose the coordinates $x, y$ in the plane $\alpha=\{z=0\}$ so that the line $y=0$ is tangent to $K_{1}^{\prime}$ and $K_{2}^{\prime}$ and so that $K^{\prime}$ is contained in the half-plane $y \geq 0$. Let $q_{1} \in K_{1}^{\prime}$ and $q_{2} \in K_{2}^{\prime}$ be such that $\left[q_{1}, q_{2}\right]$ is the shortest segment connecting $K_{1}^{\prime}$ and $K_{2}^{\prime}$. Take a point $q_{1}^{\prime} \in \partial K_{1}^{\prime}$ close to $q_{1}$ and such that the $y$-coordinate of $y_{1}^{\prime}$ is smaller than that of $q_{1}$. Consider the open arc A on $\partial K_{1}^{\prime}$ connecting $q_{1}$ and $q_{1}^{\prime}$. It is clear that $\mathrm{A} \cap C=\emptyset$.

Let $f: \mathbb{R}^{3} \longrightarrow \mathbb{R}^{3}$ be a $C^{1}$ (we can make it even $C^{\infty}$ ) diffeomorphism with $f(x)=x$ for all $x$ outside a small open set $U$ such that $q_{1} \in \bar{U}$ and $U \cap \partial K^{\prime} \subset \mathrm{A}$. We can choose $f$ so that $\widetilde{K}_{i}=f\left(K_{i}\right)=K_{i}$ for $i>1, \widetilde{K}_{1}=f\left(K_{1}\right)$ is strictly convex, and $\tilde{\nu}(f(q)) \notin \alpha$ for $q \in \mathrm{A}$ arbitrarily close to $q_{1}$. Here $\tilde{\nu}$ is the outward unit normal field to $\partial \widetilde{K}$, where $\widetilde{K}=\bigcup_{i=1}^{k_{0}} \widetilde{K}_{i}$. Clearly with this choice of $f$ for any $q \in C$ the tangent planes $T_{q}(\partial K)$ and $T_{q}(\partial \widetilde{K})$ coincide.

Notice that the non-wandering set $\widetilde{\Lambda}$ for the billiard flow $\tilde{\phi}_{t}$ in the closure $\widetilde{\Omega}$ of the exterior of $\widetilde{K}$ in $\mathbb{R}^{3}$ coincides with $\Lambda$. Indeed, let $\widetilde{\Phi}: \Sigma_{A} \longrightarrow \Lambda \cap S_{\partial \widetilde{K}}^{*}(\widetilde{\Omega})$ be the coding map for the billiard trajectories in $\widetilde{\Omega}$ and let $\tilde{z}=(\tilde{q}, \tilde{\xi}) \in S_{\partial \widetilde{K}}^{*}(\widetilde{\Omega})$ belong to $\widetilde{\Lambda}$. Then $\tilde{z}=\widetilde{\Phi}(\xi)$ for some $\xi \in \Sigma_{A}$, so $z=\Phi(\xi) \in \Lambda \cap S_{\partial K}^{*}(\Omega)$. Since any reflection point $P_{i}(\xi) \in C$, by the choice of $f$ we have that $P_{j}(\xi)$ are the successive reflection points of a billiard trajectory in $\widetilde{\Omega}$, and this must be the trajectory determined by $\widetilde{\Phi}(\xi)$. Thus, $\tilde{z}=z$, so $\tilde{z} \in \Lambda$. This argument also shows that $\Lambda \subset \widetilde{\Lambda}$, so $\widetilde{\Lambda}=\Lambda \subset S^{*}(\alpha)$. Thus, $\operatorname{dim}\left(E_{\widetilde{\Lambda}}^{u}(z)\right)=1<\operatorname{dim}\left(E^{u}(z)\right)$ for any $z \in \widetilde{\Lambda}$. However, it is clear from the construction that $S^{*}(\alpha \cap \Omega)$ is not invariant with respect to the billiard flow $\tilde{\phi}_{t}$. Moreover, it is not difficult to see that there is no two-dimensional submanifold $\tilde{\alpha}$ of $\widetilde{\Omega}$ such that $S^{*}(\tilde{\alpha})$ is $d \tilde{\phi}_{t}$-invariant and $\Lambda \subset S^{*}(\tilde{\alpha})$.

Indeed, assume such $\tilde{\alpha}$ exists; then $\tilde{\alpha}$ is a union of billiard trajectories and $C=\operatorname{pr}_{1}(\Lambda) \subset \tilde{\alpha}$. We will first show that $\tilde{\alpha}=\alpha$ outside a large disk $D$ containing $K^{\prime}$. Changing the coordinates $x, y$ in the plane $\alpha=\{z=0\}$, we may assume that the line $y=0$ is tangent to $K_{2}^{\prime}$ and $K_{3}^{\prime}$ and that $K^{\prime}$ is contained in the half-plane 
$y \geq 0$. Let $p_{2} \in K_{2}^{\prime}$ and $p_{3} \in K_{3}^{\prime}$ be such that $\left[p_{2}, p_{3}\right]$ is the shortest segment connecting $K_{2}^{\prime}$ and $K_{3}^{\prime}$. Clearly $p_{2}, p_{3} \in \tilde{\alpha}$. Since $p_{2}$ is not an isolated point in $C$, there are points in $C \cap \partial K_{2}^{\prime}$ arbitrarily close to $p_{2}$. All of them are in $\tilde{\alpha}$, so the curve $\tilde{\alpha} \cap \partial K_{2}$ is tangent to $\partial K_{2}^{\prime}$ at $p_{2}$. Similarly, the curve $\tilde{\alpha} \cap \partial K_{3}$ is tangent to $\partial K_{3}^{\prime}$ at $p_{3}$. Take a point $p_{2}^{\prime} \in \tilde{\alpha} \cap \partial K_{2}$ close to $p_{2}$ and a point $p_{3}^{\prime} \in \tilde{\alpha} \cap \partial K_{3}$ close to $p_{3}$ such that the $y$-coordinates of $p_{2}$ and $p_{3}$ are smaller than those of $p_{2}^{\prime}$ and $p_{3}^{\prime}$, respectively. Consider the $\operatorname{arc} C_{2}$ on $\tilde{\alpha} \cap \partial K_{2}$ connecting $p_{2}$ and $p_{2}^{\prime}$ and the $\operatorname{arc} C_{3}$ on $\tilde{\alpha} \cap \partial K_{3}$ connecting $p_{3}$ and $p_{3}^{\prime}$. We have $\left(p_{2}, v_{2}\right) \in \Lambda$, where $v_{2}=\left(p_{3}-p_{2}\right) /\left\|p_{3}-p_{2}\right\|$. For any $p \in C_{2}$ sufficiently close to $p_{2}$ and any $v \in S_{p}^{*}(\tilde{\alpha})$ sufficiently close to $v_{2}$, the billiard trajectory $\gamma(p, v)$ in $S^{*}(\widetilde{\Omega})$ issued from $(p, v)$ is contained in $S^{*}(\tilde{\alpha})$, so its projection in $\mathbb{R}^{3}$ is contained in $\tilde{\alpha}$. Thus, the first reflection point of $\gamma(p, v)$ belongs to $C_{3}$. That is, for any $p \in C_{2}$ sufficiently close to $p_{2}$ and any $v \in S_{p}^{*}(\tilde{\alpha})$ sufficiently close to $v_{2}$, the straight line segment ray issued from $p$ in the direction of $v$ intersects the curve $C_{3}$. Similarly, for any $q \in C_{3}$ sufficiently close to $p_{3}$ and any $w \in S_{q}^{*}(\tilde{\alpha})$ sufficiently close to $-v_{2}$, the straight line segment ray issued from $q$ in the direction of $w$ intersects the curve $C_{2}$. Replacing the curves $C_{2}$ and $C_{3}$ by shorter ones (i.e. replacing the points $p_{2}^{\prime}$ and $p_{3}^{\prime}$ by points on $C_{2}$ and $C_{3}$ closer to $p_{2}$ and $p_{3}$, respectively), we now have that for any $p \in C_{2}$ and any $q \in C_{3}$, the straight line segment $[p, q]$ lies in $\tilde{\alpha}$. This shows that $C_{2}$ and $C_{3}$ lie in the same plane, and it is clear from the choice of $C_{2}$ and $C_{3}$ that this plane is tangent to $\alpha$ at $p_{2}$, so it must coincide with $\alpha$. Moreover, the union of the segments $[p, q]$ with $p \in C_{2}$ and any $q \in C_{3}$ is part of both $\alpha$ and $\tilde{\alpha}$.

From the latter one easily derives that if $D$ is an open disk in $\alpha$ containing $K^{\prime}$, then $\alpha \backslash D \subset \tilde{\alpha}$. Since $\Lambda$ is a nowhere dense subset of $S^{*}(\Omega)$ (and $S^{*}(\widetilde{\Omega})$, as well), every point $q \in \alpha \backslash K$ can be approximated arbitrarily well by points of the form $\operatorname{pr}_{1}\left(\phi_{t}(x, \xi)\right)$, where $t>0$ and $(x, \xi) \in S^{*}(\alpha \backslash D)$. This implies $\alpha \backslash K \subset \tilde{\alpha}$, and therefore $\alpha \backslash K=\tilde{\alpha}$, which is a contradiction with the definition of $\widetilde{K}$ and the perturbation $f$. Hence there does not exist a two-dimensional submanifold $\tilde{\alpha}$ of $\widetilde{\Omega}$ such that $S^{*}(\tilde{\alpha})$ is $d \tilde{\phi}_{t}$-invariant and $\Lambda \subset S^{*}(\tilde{\alpha})$.

4.2. Geodesic flows on manifolds of negative curvature. Let $X$ be a complete (not necessarily compact) connected Riemann manifold of constant curvature $K=$ -1 and dimension $\operatorname{dim}(X)=n+1, n \geq 1$, and let $\phi_{t}: M=S^{*}(X) \longrightarrow M$ be the geodesic flow on the unit cosphere bundle of $X$. According to a classical result of Killing and Hopf any such $X$ is a hyperbolic manifold, i.e. $X$ is isometric to $\mathbb{H}^{n+1} / \Gamma$, where

$$
\mathbb{H}^{n+1}=\left\{\left(x_{1}, \ldots, x_{n+1}\right) \in \mathbb{R}^{n+1}: x_{1}>0\right\}
$$

is the upper half-space in $\mathbb{R}^{n+1}$ with the Poincaré metric $d s^{2}(x)=\frac{1}{x_{1}^{2}}\left(d x_{1}^{2}+\ldots+\right.$ $\left.d x_{n+1}^{2}\right)$ and $\Gamma$ is a Kleinian group, i.e. a discrete group of isometries (Möbius transformations) acting freely and discontinuously on $\mathbb{H}^{n+1}$. See e.g. Ratc for basic information on hyperbolic manifolds. Given a hyperbolic manifold $X=\mathbb{H}^{n+1} / \Gamma$, the limit set $L(\Gamma)$ is defined as the set of accumulation points of all $\Gamma$ orbits in $\overline{\partial \mathbb{H}^{n+1}}$, the topological closure of $\partial \mathbb{H}^{n+1}=\{0\} \times \mathbb{R}^{n}$ including $\infty$.

Throughout this subsection we will assume that $\Gamma$ is torsion-free and finitely generated (then $\Gamma$ is called geometrically finite) and non-elementary; i.e. $L(\Gamma)$ is infinite (then $L(\Gamma)$ is a closed non-empty nowhere dense subset of $\partial \overline{\mathbb{H}^{n+1}}$ without isolated points; see e.g. Section 12.1 in [Ratc]). A geometrically finite Kleinian 
group with no parabolic elements is called convex cocompact. If $X$ is compact, then $\Gamma$ is called a cocompact lattice.

The non-wandering set $\Lambda$ of $\varphi_{t}: M \longrightarrow M$ (also known as the convex core of $\left.X=\mathbb{H}^{n+1} / \Gamma\right)$ is the image in $M$ of the set of all points of $S^{*}\left(\mathbb{H}^{n+1}\right)$ generating geodesics with end points in $L(\Gamma)$. When $\Gamma$ is convex cocompact, the non-wandering set $\Lambda$ is compact.

Notice that the class of hyperbolic manifolds $X=\mathbb{H}^{n+1} / \Gamma$, with $\Gamma$ a nonelementary convex cocompact Kleinian group, contains all classical and non-classical Schottky manifolds (cf. e.g. Section 12.1 in Ratc). In this case the (un)stable laminations of the geodesic flow over $\Lambda$ are always $C^{1}$ (in fact $C^{\infty}$ ).

Example 4.2. Consider the case $n=2$ and let $\mathbb{H}^{3} / \Gamma$ be a hyperbolic manifold generated by a non-elementary convex cocompact Kleinian group $\Gamma$ with an "Apolonian packing" limit set $L(\Gamma)$ (see e.g. $[\mathrm{Su}]$ ). Then $L(\Gamma)$ is a subset of $\mathbb{R}^{2}$ such that for every $x \in L(\Gamma)$ the set of tangent vectors to $L(\Gamma)$ at $x$ is one-dimensional, while $L(\Gamma) \cap U$ is not contained in a one-dimensional submanifold of $\mathbb{R}^{2}$ for any open neighbourhood $U$ of $x$ in $\mathbb{R}^{2}$. Given $z \in \Lambda$, the local unstable manifold $W_{\epsilon}^{u}(z)$ is given by the projection into $\mathbb{H}^{3} / \Gamma$ of the outward normal field to a (part of) a horosphere $S$ in $\mathbb{H}^{3}$. The latter are just spheres in $\mathbb{H}^{3}$ tangent to $\partial \mathbb{H}^{3}$ or planes in the interior of $\mathbb{H}^{3}$ parallel to $\partial \mathbb{H}^{3}$. Any $z^{\prime} \in \Lambda \cap W_{\epsilon}^{u}(z)$ can be identified with the point $x^{\prime} \in L(\Gamma)$, which is just the limit at $+\infty$ of the geodesic determined by $z^{\prime}$. It then follows that the set of tangent vectors in $E^{u}(z)$ to $\Lambda$ at $z$ is one-dimensional, while $\Lambda \cap U$ is not contained in a one-dimensional submanifold of $W_{\epsilon}^{u}(z)$ for any $\epsilon>0$, i.e. $k^{u}=2$.

\section{REFERENCES}

[B] R. Bowen, Symbolic dynamics for hyperbolic flows, Amer. J. Math. 95 (1973), 429-460. MR 0339281 (49:4041)

[Ch] N. Chernov, Invariant measures for hyperbolic dynamical systems, in: Handbook of Dynamical Systems, ed. by A. Katok and B. Hasselblatt, Vol. 1A, pp. 321-407, North-Holland, Amsterdam, 2002. MR1928521 (2003g:37047)

[D] D. Dolgopyat, On decay of correlations in Anosov flows, Ann. of Math. (2) 147 (1998), 357-390. MR 1626749 (99g:58073)

[GP] V. Guillemin and A. Polack, Differential topology, Prentice Hall, New Jersey, 1974. MR0348781 (50:1276)

[Ha] B. Hasselblatt, Regularity of the Anosov splitting and of horospheric foliations, Ergod. Th. \& Dynam. Sys. 14 (1994), 645-666. MR.1304137 (95j:58130)

[Ka] M. Kapovich, Kleinian groups in higher dimensions, Progress in Mathematics, Vol. 265, Birkhäuser Basel, 2008, 485-562. MR2402415 (2009g:30043)

$[\mathrm{KH}]$ A. Katok and B. Hasselblatt, Introduction to the Modern Theory of Dynamical Systems, Cambridge Univ. Press, Cambridge, 1995. MR1326374 (96c:58055)

[M] B. Malgrange, Ideals of differentiable functions, Oxford University Press, 1966. MR0212575(35:3446)

[PSW] C. Pugh, M. Shub and A. Wilkinson, Hölder foliations, Duke Math. J. 86 (1997), 517-546; Correction: Duke Math. J. 105 (2000), 105-106. MR.1432307 (97m:58155)] MR.1788044 (2001h:37057)

[Ratc] J. G. Ratcliffe, Foundations of hyperbolic manifolds, Springer-Verlag, New York, 1994. MR 1299730 (95j:57011)

[St1] L. Stoyanov, Exponential instability and entropy for a class of dispersing billiards, Ergod. Th. \& Dynam. Sys. 19 (1999), 201-226. MR.1677157(99m:58149)

[St2] L. Stoyanov, Spectra of Ruelle transfer operators for Axiom A flows, Nonlinearity 24 (2011), 1089-1120. 
[St3] L. Stoyanov, Non-integrability of open billiard flows and Dolgopyat type estimates, Ergod. Th. \& Dynam. Sys., to appear, doi:10.1017/S0143385710000933.

[St4] L. Stoyanov, Pinching conditions, linearization and regularity of Axiom A flows, preprint (arXiv: math.DS:1010.1594).

[Su] D. Sullivan, Entropy, Hausdorff measures old and new, and limit sets of geometrically finite Kleinian groups, Acta Math. 153 (1984), 259-278. MR766265|(86c:58093)

School of Mathematics, University of Western Australia, Crawley, WA 6009, AusTRALIA

E-mail address: stoyanov@maths.uwa.edu.au 